\title{
Attentional templates regulate competitive interactions among attended visual objects
}

\author{
JEFFREY R. W. MounTS \\ State University of New York, Geneseo, New York \\ JASON S. MCCARLEY \\ University of Illinois at Urbana-Champaign, Urbana, Illinois \\ AND \\ ANDREW M. TERECH \\ State University of New York, Geneseo, New York
}

\begin{abstract}
In two experiments, we examined the mechanisms responsible for creating a zone of interference surrounding an attended visual object (see, e.g., Mounts \& Gavett, 2004). In Experiment 1, the similarity between attended stimuli and noise items was manipulated in order to contrast an account based on competitive interactions between attended items with an account based on inefficient filtering of unattended stimuli. Consistent with the competitive interaction account, the data revealed that similarity between attended items increased the strength of localized interference, whereas similarity of noise items to the attended stimuli did not. Experiment 2 showed that the interference observed between attended items was determined by their match to attentional templates.
\end{abstract}

The mechanisms of visual selective attention have been variously described as space based (e.g., Eriksen \& Hoffman, 1973; Posner, 1980), object based (e.g., Duncan, 1984; Egly, Driver, \& Rafal, 1994), and feature based (e.g., J. Lu \& Itti, 2005; Saenz, Buracas, \& Boynton, 2002; Treue \& Martínez Trujillo, 1999). Recently, these distinct models have begun to be subsumed under the conceptualization of attention as a competition among objects for representation within the visual system (Cave, 1999; Deco \& Rolls, 2004; Desimone \& Duncan, 1995; Luck, Girelli, McDermott, \& Ford, 1997; Vecera, 2000). The competitive interaction framework proposes that stimulus objects compete to be represented by visually selective neurons within the extrastriate cortex. The need for competition is imposed by the neurophysiology of the visual cortex. Receptive fields (RFs) are small in V1, the earliest cortical visual area, but grow larger through the extrastriate cortex, achieving sizes large enough to encompass two or more objects simultaneously. The result is that a single neuron's response train can conflate the properties of multiple objects (Luck \& Beach, 1998; Reynolds \& Desimone, 1999; Treisman, 1996). One solution for this problem is to employ a form of noise exclusion (cf. Dosher \& Lu, 2000; Z.-L. Lu \& Dosher, 1998, 2004), filtering signals as they propagate from early to later visual areas, so that the input reaching a given high-level cell arises from only a single stimulus object. The neuron thus becomes temporarily dedicated to the representation of a single object (Moran \& Desimone, 1985; Reynolds \& Desimone, 2003).
Gating of information to higher level cells is controlled by interactions between neurons at multiple levels of representation. To account for increases in RF size across levels of the visual cortex, many competitive interaction models posit a pyramidal processing architecture wherein projections from pools of adjacent neurons converge from one level of representation to the next (e.g., Cave, 1999; Deco \& Rolls, 2004; Tsotsos et al., 1995). Competition between objects, driven bottom up by stimulus salience and top down by attentional set (Desimone \& Duncan, 1995; Mounts, 2005; Mounts \& Gavett, 2004), is instantiated by lateral inhibitory interactions between neurons within each level of the representational hierarchy. During exposure to a visual scene, then, signals from stimulus objects are initially fed forward from lower to higher levels of representation. Following this initial wave of widespread activation, however, competitive processes occurring within each level of representation, and integrated by forward and back projections between levels, begin to prune the signals propagating from lower to higher levels until eventually a single representation (e.g., Hamker, 2004; Tsotsos et al., 1995), or at best a small number of representations (e.g., Bundesen, Habekost, \& Kyllingsbæk, 2005), emerges at the highest level of coding. ${ }^{1}$

Because of the changes in RF size that occur through the processing hierarchy, the strength of the competitive interactions between stimuli is inversely related to their spatial separation. At lower levels of the representational

J. R.W. Mounts, mounts@geneseo.edu 
pyramid, where RF sizes are smaller, interactions extend over restricted regions of the visual field and competition occurs only between objects that are near one another. At progressively higher levels of representation, where RFs become larger and interactions thus extend over increasingly greater distances, mutual interference emerges between objects that are widely separated. The more distant two objects are within the visual field, in other words, the further they can propagate through the representational hierarchy before coming into competition with one another. Functional imaging data corroborate this claim, showing that the spatial extent of suppressive interactions between objects increases from early to later regions of the visual cortex as RFs become larger (Kastner et al., 2001). Theoretical considerations, as well as neurophysiological and neuroimaging data, thus imply that the amount of information that can be extracted from multiple stimuli in parallel increases with the separation between those stimuli. Consistent with this prediction, psychophysical studies have shown that attentional processing of one item within the visual field can degrade processing of surrounding stimuli and that the strength of this interference diminishes with increased separation between the items. Same-different judgments of cued stimulus forms, for instance, become increasingly poorer as the cued items are moved nearer one another in the visual field (Cutzu \& Tsotsos, 2003; McCarley, Mounts, \& Kramer, 2004). Similarly, accuracy in identifying each of two items within a pair of cued stimulus characters is highest when the attended objects are distant within the visual field and becomes poorer as they are moved closer together (Bahcall \& Kowler, 1999; Kristjánsson \& Nakayama, 2002; McCarley \& Mounts, 2007). Perceptual processing of a probe stimulus, as another example, is degraded in the neighborhood of an attention-capturing distractor (Caputo \& Guerra, 1998; Mounts, 2000a, 2000b; Theeuwes, Kramer, \& Kingstone, 2004; Turatto \& Galfano, 2001) or an attentional target stimulus (Cave \& Zimmerman, 1997). Explanations of such effects in terms of purely sensory-level interactions have been ruled out. For instance, Bahcall and Kowler (1999) and Mounts and Gavett (2004) observed this surround interference even when the cues signaling the tobe-attended items were removed $500 \mathrm{msec}$ prior to the imperative display, so that the sensory properties of the imperative displays themselves were held constant across changes in separation between the attended items. McCarley et al. (2004), similarly, found that spatial interference was eliminated when observers were required to attend to only one, rather than both, of two colored items present within an array of distractors. The effect can thus be described as localized attentional interference.

\section{Evaluating the Source of Localized Attentional Interference}

As has been outlined above, the observation of localized attentional interference is consistent with the competitive interaction framework, which predicts spatially mediated interference when observers attempt to attend to and identify multiple stimuli. However, the competitive interaction model allows at least two potential causes of this effect. One possibility is that the performance decrement observed at small separations is a direct result of reciprocal competitive interactions between the attended stimuli. As the two attended items are moved nearer one another, that is, each may suppress the other more strongly, leading to an increase in the strength of mutual degradation. (Experiment 2 in the present report examines the nature of the interactions between attended stimuli more closely.) Thus, according to this account, localized interference arises due to competitive interactions between the two targets themselves. Call this the reciprocal interference account. An alternative possibility is that localized interference is the result of impaired filtering when attended items fall in close proximity to one another. In particular, it is possible that the demand to select two items in close spatial proximity may require that the lateral inhibitory mechanisms that normally drive target selection be relaxed. This would occur because the items that constitute noise for one taskrelevant item - noise that ordinarily is suppressed — will include the second task-relevant stimulus, which also requires analysis. Relaxing local inhibitory interactions in order to accumulate information from both targets, however, would decrease the extent to which surrounding visual noise is suppressed, leading to an increase in interference from surrounding noise items. Only as the separation between attended items was increased would localized inhibitory processes gradually become able to function normally in suppressing surrounding items. In this view, localized attentional interference reflects an increase in the disruptive effects of task-irrelevant noise items when two stimuli in close spatial proximity must be identified. Call this the relaxed-filtering account.

How can these distinct accounts be tested? In the displays typically used to study localized attentional interference, the primary source of external noise is the filler stimuli among which targets are embedded. The purpose of these fillers is to control for sensory interactions between stimuli by ensuring that stimulus density remains constant as the separation between attended items varies. According to the relaxed-filtering account of localized attentional interference, it is the failure to suppress these fillers that disrupts processing when attended items are in close proximity to one another. By manipulating the characteristics of the filler stimuli, it may therefore be possible to manipulate the strength of localized interference between attended items. According to the reciprocal interference account, in contrast, it is the inhibitory signals between attended items themselves that degrade performance as spatial separation decreases. Characteristics of the filler stimuli should, therefore, have no effect on the magnitude of localized interference, but characteristics of the attended stimuli should. In Experiment 1, we discriminated between these two alternatives by manipulating (1) the similarity between the objects within a pair of attended items and (2) the similarity between an attended target item and the set of task-irrelevant filler items embedding the attended stimuli. To preview, the data suggest that the strength of localized attentional interference is determined by the characteristics of the attended items themselves, consistent with the reciprocal interference ac- 
count, and not by characteristics of the unattended filler items. In Experiment 2, we examined more closely the mechanisms by which the stimulus characteristics that influence the reciprocal interference between attended items produce localized interference.

\section{EXPERIMENT 1}

In Experiment 1, color was used to cue a pair of potential target objects among an array of gray filler objects. One of the colored objects, the target, was a $\mathrm{T}$ that appeared either in its canonical orientation or rotated $180^{\circ}$. The other colored object, the decoy, was a non-T shape (see below). Spatial separation between the colored objects (i.e., the target and the decoy) varied across trials. The participants' task was to judge the orientation of the target. Because it was unknown a priori which of the two colored objects would be the target, it was necessary to attend to both colored objects. In light of previous data showing localized interference in tasks requiring observers to divide attention between multiple cued objects (e.g., Bahcall \& Kowler, 1999; McCarley et al., 2004; Mounts \& Gavett, 2004), performance was therefore expected to decline as the separation between the color-cued objects decreased. To examine the contributions of reciprocal interactions and noise suppression to localized interference, two additional stimulus characteristics were manipulated orthogonal to the separation between attended items: the similarity of the decoy shape to the target shape and the similarity of the filler shapes to the target shape. The similarity manipulations were used to identify which class of nontarget items in the display - the decoy or the fillerswas responsible for disrupted target processing in localized attentional interference. In general, we expected that increased similarity between an interference-producing item - a decoy or a filler - and the target would degrade target identification. In Experiment 2, we explored the specific mechanisms by which this might occur.

If localized interference in the present task results from reciprocal interference between the attended items themselves, target-decoy similarity should interact with the separation between the cued items, with high-similarity decoys creating greater interference at small separations than do low-similarity decoys. If the fillers are the source of localized attentional interference, as suggested by the relaxed-filtering account, target-filler similarity should interact with the separation between the attended items, producing greater interference at small values of separation when the fillers and the target are highly similar.

\section{Method}

Subjects. Twenty students from the State University of New York at Geneseo participated in the experiment in exchange for course credit. All the subjects reported normal or corrected-to-normal visual acuity and were screened for normal color vision, using the Ishihara color blindness test (1989).

Apparatus and Stimuli. The stimuli were presented on a 19-in. monitor with a resolution of $1,024 \times 768$ pixels and a frame rate of $75 \mathrm{~Hz}$. The subjects were seated $57 \mathrm{~cm}$ from the monitor and were stabilized through the use of a chin-and-forehead rest. Responses were made using the right and left index fingers on a PST Serial
Response Box 300 (Psychology Software Tools). All the sessions were conducted in a darkened booth.

The stimulus items were Ls, Cs, and Ts in one of four orientations: rotated $0^{\circ}, 90^{\circ}, 180^{\circ}$, or $270^{\circ}$ from their canonical orientations. Each item subtended $1.1^{\circ} \times 1.1^{\circ}$ of visual angle and was drawn in stroke $0.2^{\circ}$ in width. The $\mathrm{C}$ stimuli were circles with an appropriately positioned gap that subtended $0.25^{\circ}$ of visual angle. The filler items were gray $\left(x=.27, y=.28\right.$; luminance: $\left.14.88 \mathrm{~cd} / \mathrm{m}^{2}\right)$. One of the colored items on each trial (either the target or the decoy) was red $(x=.36$, $y=.27$; luminance: $\left.15.38 \mathrm{~cd} / \mathrm{m}^{2}\right)$, and the other was green $(x=$ .23, $y=.35$; luminance: $15.45 \mathrm{~cd} / \mathrm{m}^{2}$ ). The assignment of colors to target and decoy items was determined randomly on each trial. All the stimuli were presented on a black background $(x=.27, y=.28$; luminance: $1.90 \mathrm{~cd} / \mathrm{m}^{2}$ )

Conditions and Procedure. On each trial, exactly one of the two colored items was a T. This was the target. The subjects' task was to judge whether the colored $\mathrm{T}$ was canonically oriented (left-key response) or rotated $180^{\circ}$ (right-key response). Note that because the color of the target was unpredictable from trial to trial, the subjects were required to attend to both of the colored items in order to determine which was the target. Targets appeared equally often at each of the 12 stimulus positions, which were equally spaced along an imaginary circle with a radius of $5.2^{\circ}$. The other colored item (the decoy) was located either one position (adjacent to the target), three positions (two intervening fillers), or five positions (four intervening fillers) from the target. This created three levels of attended item separation, corresponding to angular separations of $30^{\circ}, 90^{\circ}$, or $150^{\circ}$. The gray fillers could be (1) Ts oriented canonically or rotated $180^{\circ}$ from canonical, (2) Ts rotated $90^{\circ}$ or $270^{\circ}$ from canonical, (3) Ls rotated $0^{\circ}, 90^{\circ}, 180^{\circ}$, or $270^{\circ}$ from canonical, or (4) Cs rotated $0^{\circ}, 90^{\circ}, 180^{\circ}$, or $270^{\circ}$ from canonical. This produced four levels of target-filler similarity. ${ }^{2}$ Finally, the decoy item (the colored nontarget) could assume one of two forms: either an $\mathrm{L}$ rotated $0^{\circ}, 90^{\circ}, 180^{\circ}$, or $270^{\circ}$ from canonical or a $\mathrm{C}$ rotated $0^{\circ}, 90^{\circ}, 180^{\circ}$, or $270^{\circ}$ from canonical. This resulted in two levels of target-decoy similarity. The three levels of attended item separation, four levels of target-filler similarity, and two levels of target-decoy similarity were completely counterbalanced, along with the two target values $\left(0^{\circ}\right.$ or $\left.180^{\circ}\right)$ and 12 possible target locations, yielding 576 experimental trials. On each trial, the colors of the target and decoy items were randomly assigned (one as red, the other as green). Figure 1 shows a sample stimulus display with an attended item separation of one, $\mathrm{L}$ fillers, and a $\mathrm{C}$ decoy.

Each trial began with a fixation cross in the center of a blank screen. The subjects were instructed to maintain fixation on the cross throughout the trial. After $500 \mathrm{msec}$, the stimulus array was presented for an exposure duration of $200 \mathrm{msec}$, followed by a blank screen. The subjects were instructed to respond as quickly as possible as to the orientation of the target while maintaining accuracy of $90 \%$. Incorrect responses were signaled by an $X$ in the center of the screen. Each subject completed 24 randomly chosen practice trials, followed by 12 blocks of 48 experimental trials. Each session lasted approximately $40 \mathrm{~min}$.

\section{Results and Discussion}

Data from 3 subjects were replaced because of error rates that exceeded $15 \%$. Outliers in the reaction time (RT) data were trimmed by excluding trials in which responses were faster than $300 \mathrm{msec}$ or more than three standard deviations above the mean RT for a given subject in a given condition. This led to the rejection of fewer than $2 \%$ of the trials. RTs from incorrect trials were excluded from the RT analysis, resulting in a loss of fewer than $6 \%$ of the trials.

RTs were analyzed using a $2 \times 4 \times 3$ ANOVA, with decoy form (two levels), filler form (four levels), and attended item separation (three levels) as within-subjects 


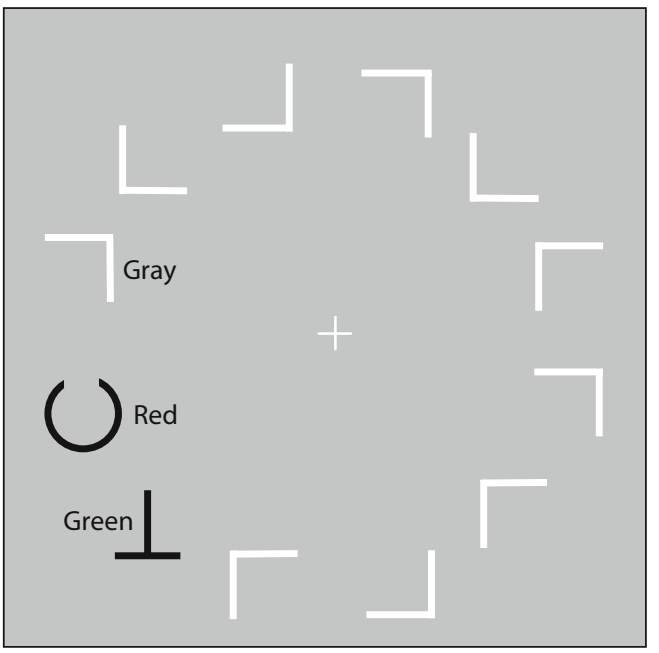

Figure 1. An example stimulus display from Experiment 1. Light items represent the gray filler stimuli. Dark items represent the target (T) and decoy items (one red, the other green).

factors. The top panel in Figure 2 shows the mean RT across observers for each decoy/filler condition as a function of attended item separation. Main effects were observed for all three factors [decoy form, $F(1,19)=$ $135.28, p<.001$; filler form, $F(3,57)=17.88, p<.001$; attended item separation, $F(2,38)=5.24, p<.01]$. The observation of main effects of decoy and filler form demonstrated that the increased similarity disrupted target performance, whereas the main effect of attended item separation confirmed that the dual-cue procedure yielded localized attentional interference, as has been observed in other studies. We next will examine the interactions involving these factors. Recall that the different explanations of localized attentional interference predict different patterns of interactions between attended item separation and decoy type and filler type. Consistent with the idea that localized attentional interference reflects interference between the decoy and the target item, as predicted by the reciprocal interference account, the data revealed an interaction between decoy type and attended item separation $[F(2,38)=7.60, p<.005]$, with $\mathrm{L}$ decoys producing greater localized interference than that produced by $\mathrm{C}$ decoys. The relaxed-filtering account posits that filler items interfere more with the target as the separation between the attended items is decreased. This account predicts an interaction between filler form and separation, an effect that was not observed $[F(6,114)<1]$. So, although increased filler similarity generally disrupted target processing, this effect was independent of the processes resulting in the attended item separation effects (i.e., localized attentional interference). Mechanisms by which the fillers might influence target processing will be discussed below in the General Discussion section.

Error rate data are shown in the bottom panel of Figure 2. The pattern of errors mimicked that seen in RTs, arguing against a speed-accuracy trade-off. A similar ANOVA on the error data revealed main effects of decoy type $[F(1,19)=23.36, p<.001]$ and filler type
$[F(3,57)=7.43, p<.001]$, whereas the main effect of separation did not reach statistical significance $[F(2,38)=$ $2.62, p=.08]$. As in the RT data, the only interaction to reach significance was the two-way interaction between decoy type and separation $[F(2,38)=3.54, p<.05$; all other $F \mathrm{~s}<1]$.

In sum, the data demonstrate that localized attentional interference is modulated by target-decoy similarity but is unaffected by target-filler similarity, consistent with the reciprocal interference account presented above and contrary to the relaxed-filtering account. However, this does not explain precisely how similarity modulates the interference between attended items. In Experiment 2, we addressed this question.

\section{EXPERIMENT 2}

Experiment 1 showed that the magnitude of localized attentional interference in a target identification task was increased when the members of a pair of attended items were perceptually similar. Previous research has suggested at least two ways by which this might happen. One possibility, proposed by Estes (1972), is that similarity-driven interference might arise from limits in the availability of feature-selective processing channels. In this view, channels tuned to a given spatial region and a given range of feature values are responsible for the initial encoding of visual stimuli. Spatially proximal stimuli sharing the same features would be processed by a common set of channels. Limitations in the number of channels available would require that stimuli share processing capacity, producing a degradation of the perceptual representation. When stimuli share no features and can therefore be processed by distinct perceptual channels, processing capacity need not be divided among the stimuli. Within the competitive interaction framework, the perceptual channels in question would presumably correspond to neurons selectively tuned for stimulus features such as color, orientation, or form, nested within the set of cells selectively responsive to a given location. Call this the channel interaction account of decoy-target similarity effects.

An alternative possibility, noted by Pashler (1987), is that similarity between target and decoy items might degrade performance by increasing the degree to which the decoys attract attention, making them more difficult to suppress or inhibit (see also Kim \& Cave, 2001). To select a stimulus on the basis of prespecified target properties, an observer must establish a target template, a mental representation of the desired stimulus. Attentional weights can then be assigned to stimuli on the basis of the degree to which they match the template (Bundesen et al., 2005; Duncan \& Humphreys, 1989). High weights are assigned to stimuli whose features most closely match those of the template, and lower weights are assigned to stimuli that match the template more poorly. These attentional weights then determine the strength of top-down biasing applied to each stimulus. Target-decoy similarity modulates interference, then, because decoys that more closely match the target template are assigned higher attentional weights and, therefore, compete more strongly with the target for 

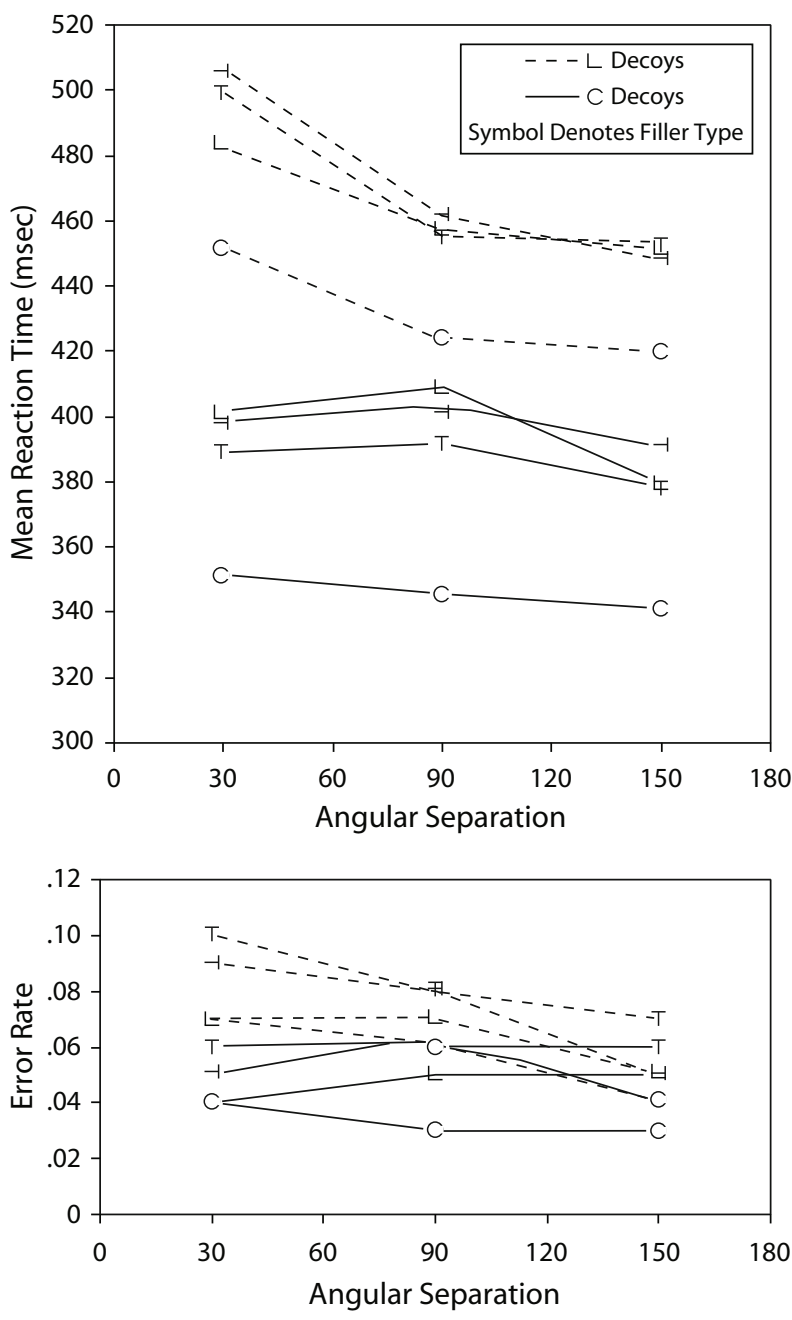

Figure 2. Mean correct reaction times (top) and error rates (bottom) for Experiment 1 as a function of filler form, decoy form, and attended item separation. Symbols represent filler form. Dashed lines indicate $\mathrm{L}$ decoys, and solid lines indicate $\mathrm{C}$ decoys. The abscissa shows the angular separation between the target and the decoy items.

perceptual resources (Desimone \& Duncan, 1995; Mounts \& Gavett, 2004; Mounts \& Tomaselli, 2005). Call this the attentional weight account of the target-decoy similarity effects seen in the first experiment.

As has been noted, both the channel interaction and the attentional weight accounts predict that high similarity between the target and the decoy items in a task such as that in Experiment 1 will exacerbate interference. They differ, however, in that the channel interaction hypothesis holds that interference is modulated by the similarity between the decoy and the target that is present within a given display, whereas the attentional weight hypothesis holds that interference is modulated more specifically by the similarity between the decoy and the target template(s). The two accounts can, therefore, be experimentally distinguished on the basis of the predictions that they make under circumstances in which the observer is required to simultaneously maintain attentional templates for two dis- similar target shapes, either of which can appear on any trial. The channel interaction account holds that interference will be high only if the decoy is similar to the target stimulus that is presented on a given trial. The attentional weight account, in contrast, predicts that interference will be high if the decoy is similar to either target template, even if it is dissimilar to the target that is actually present within a given display.

A study by Pashler (1987) illustrates this reasoning. Data from Pashler's first experiment revealed that performance in a visual search task is modulated by targetdistractor similarity, so that RTs increase with the proportion of distractors that are similar to the target. The interactive channels account ascribes this effect to bottomup perceptual interference between items processed by a common set of channels. However, Pashler noted that the effects of similarity could also reflect attention-driven interference, resulting because the distractors similar to the target required greater analysis before they could be rejected and excluded from further processing (e.g., Hoffman, 1978). To discriminate between these two accounts, Pashler (1987) changed the target set to include two possible targets and expanded the set of potential distractors to include items similar to each of the target values. Thus, the "similar" distractors could be similar to either the presented or the nonpresented target shape on each trial. The interactive channels account predicts that only distractors similar to the presented target should interfere with processing, whereas the target template account predicts comparable interference in both cases. The results strongly supported the latter conclusion: Distractors similar to the nonpresented target disrupted performance as much as did distractors similar to the target that was actually present.

Experiment 1 of the present study revealed greater localized interference when the target and the decoy were similar than when they were dissimilar. However, the results did not distinguish between the interactive channel and the attentional weight accounts of this effect. To discriminate between these possibilities, Experiment 2 adopted the logic of Pashler (1987), modifying the procedure of the present Experiment 1 to include one target form similar to the L decoy (T) and one target form similar to the $\mathrm{C}$ decoy $(\mathrm{O})$. The displays were similar to those in the first experiment, with two colored items embedded among an array of gray fillers. The observers' task was to judge whether either of the colored stimuli was a target. Here, however, the target item could be either a $\mathrm{T}$ or an $\mathrm{O}$. If the attentional weight account of the results from Experiment 1 is correct, the inclusion of the O-shaped targets should lead to an increase in the attentional salience of $\mathrm{C}$ decoys in the present experiment. Importantly, this change should hold even on trials in which the target is a T. The magnitude of interference produced by $\mathrm{C}$ decoys with a $\mathrm{T}$ target should, therefore, be equivalent to that produced by $\mathrm{L}$ decoys. Likewise, the effect of $\mathrm{L}$ decoys on the processing of an $\mathrm{O}$ target should be equivalent to the effect of $\mathrm{C}$ decoys.

The use of two perceptually distinct targets and their accompanying similar decoys necessitated a change in 
the task used in Experiment 1. In order to avoid flanker compatibility effects that might arise from each of the decoys' being more perceptually similar to one of the target alternatives (e.g., Yeh \& Eriksen, 1984), a go/no-go task was used. More specifically, the observers were asked to respond with a keypress when a target $(\mathrm{O}$ or $\mathrm{T})$ was present and to withhold the keypress otherwise.

\section{Method}

Subjects, Stimuli, and Apparatus. Twenty students from the State University of New York at Geneseo participated in exchange for course credit. All the subjects reported normal or correctedto-normal acuity and were screened for normal color vision as in Experiment 1. The apparatus was identical to that used in Experiment 1 . The only change to the stimuli involved the addition of $\mathrm{O}$ stimuli, which were identical to the $\mathrm{C}$ stimuli used in Experiment 1, but with no gap.

Conditions and Procedure. A dual-cue task was used, as in Experiment 1 , with 2 colored items embedded in an array of 10 gray filler items. On target-present trials, one of the colored items was a target shape ( $\mathrm{T}$ or $\mathrm{O})$ and the other was a decoy shape $(\mathrm{L}$ or $\mathrm{C}$, rotated $0^{\circ}, 90^{\circ}, 180^{\circ}$, or $270^{\circ}$ from canonical). On target-absent trials, both colored items were decoy shapes.

On target-present trials, the value of the target shape ( $\mathrm{T}$ or $\mathrm{O}$ ) was combined factorially with the value of the decoy shape ( $\mathrm{L}$ or C), creating four different target-decoy pairs (T-L, T-C, O-L, and $\mathrm{O}-\mathrm{C}$ ). The two color-cued items (target and decoy) could be one, three, or five positions apart (angular separation of $30^{\circ}, 90^{\circ}$, or $150^{\circ}$ ), as in Experiment 1. As is shown in Figure 3, the stimuli were arranged in alternating pairs of similar items ( $\mathrm{Ts} / \mathrm{Ls}$ and $\mathrm{Cs} / \mathrm{Os})$. As a result, the target item, whether it was a $\mathrm{T}$ or an $\mathrm{O}$, was always flanked by an $\mathrm{L}$ on one side and a $\mathrm{C}$ on the other side, regardless of target-decoy separation. In other words, the target was always flanked by one similar and one dissimilar item.

The subjects completed 36 randomly chosen practice trials, followed by 12 blocks of 36 trials, for a total of 432 experimental trials. Targets were present on 288 of the experimental trials (go trials) and absent on the remaining 144 (no-go trials). The 288 go trials were created by completely counterbalancing the two possible target

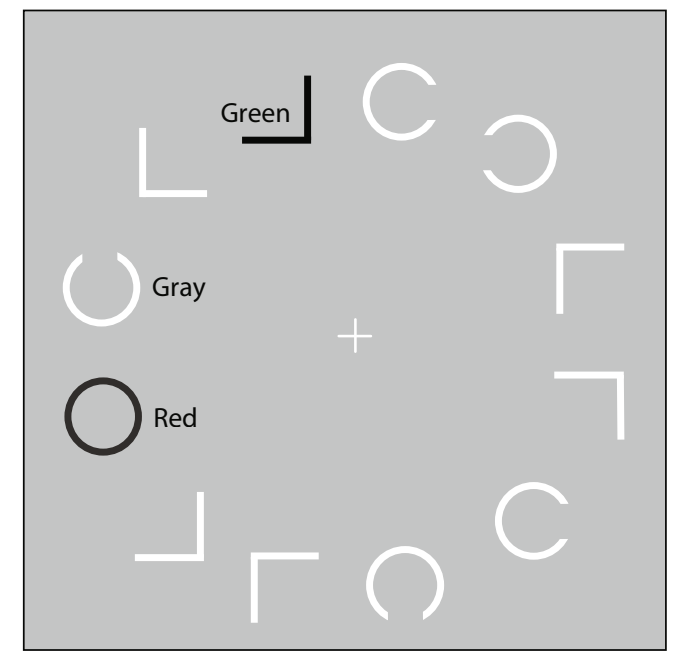

Figure 3. An example stimulus display from Experiment 2. The light items represent the gray filler items, and the dark items represent the color-cued items (one red, one green). On go trials, one of the colored items was the target (a $T$ or an $O$ ), and the other was the decoy (an $\mathrm{L}$ or a $\mathrm{C}$ ). On no-go trials, both colored items were decoys (one an $\mathrm{L}$, the other a $\mathrm{C}$ ). forms ( $\mathrm{T}$ or $\mathrm{O}$ ), the color of the target (red or green), the two possible decoy forms ( $\mathrm{L}$ or $\mathrm{C}$ ), the 12 possible target locations, and the three attended item separations $\left(30^{\circ}, 90^{\circ}\right.$, or $\left.150^{\circ}\right)$. The 144 no-go trials (in which two decoy shapes were presented) counterbalanced the values of the two decoys ( $\mathrm{L}$ or $\mathrm{C}$ ), their locations, and their angular separation $\left(30^{\circ}, 90^{\circ}\right.$, or $\left.150^{\circ}\right)$.

Each trial began with a fixation cross at the center of a blank screen. The subjects were instructed to maintain fixation on the cross throughout the trial. After $500 \mathrm{msec}$, the stimulus array was presented for an exposure duration of $200 \mathrm{msec}$, followed by a blank screen. The subjects' task was to identify whether one of the colored items was a $\mathrm{T}$ or an $\mathrm{O}$. The subjects were instructed to respond by pressing the center button on the response box if a target was present and to withhold a response otherwise. If no response was detected within $1,200 \mathrm{msec}$ after the onset of the trial, the trial was terminated, and the experiment advanced to the next trial. The subjects were instructed to respond as quickly as possible while maintaining $90 \%$ accuracy. Errors of both omission and commission were signaled by a red $\mathrm{X}$ in the center of the screen. The entire experiment lasted approximately $30 \mathrm{~min}$.

\section{Results and Discussion}

The data from 6 subjects were replaced because of miss and/or false alarm rates that exceeded $15 \%$. Inclusion of the data from these subjects did not alter the pattern of results observed. A trimming procedure similar to that in Experiment 1 was used, resulting in a loss of fewer than $2.1 \%$ of the trials.

Mean RTs for correct go trials (shown in the top panel of Figure 4) were submitted to a $2 \times 2 \times 3$ ANOVA, with target form (T or O), target-decoy similarity (high or low), and attended item separation $\left(30^{\circ}, 90^{\circ}\right.$, or $\left.150^{\circ}\right)$ as withinsubjects factors. The main effect of target form did not reach significance $[F(1,19)=2.22$, n.s.], nor did the main effect of target-decoy similarity $[F(1,19)=2.91$, n.s. $]$. The main effect of attended item separation was statistically significant $[F(2,38)=39.00, p<.001]$. The twoway interaction between target form and target-decoy similarity was reliable $[F(1,19)=5.17, p<.05]$, whereas the interaction between target form and separation was $\operatorname{not}[F(2,38)=2.60$, n.s. $]$. The critical interaction between target-decoy similarity and separation was not reliable $(F<1)$, suggesting that the effects of separation did not depend on target-decoy similarity, a result consistent with the attentional weight account of the similarity effects seen in Experiment 1 and inconsistent with the channel interaction account. Finally, the three way interaction between target form, target-decoy similarity, and separation was nonreliable $[F(2,38)=1.38$, n.s.].

To more fully test the conclusion that target-decoy similarity did not influence the degree of localized interference, separate $2 \times 3$ ANOVAs were run for each target form ( $\mathrm{T}$ and $\mathrm{O}$ ), with target-decoy similarity and separation as within-subjects factors. As has been noted, the channel interaction model predicts that localized attentional interference should be modulated by targetdecoy similarity (increased interference for $\mathrm{O}-\mathrm{C}$ and $\mathrm{T}-\mathrm{L}$ pairings), whereas the attentional weight account predicts comparable interference for all target-decoy pairings. Support for a channel interaction model in the present analyses would, therefore, come from significant interactions of target-decoy similarity and separation. For the $\mathrm{T}$ 

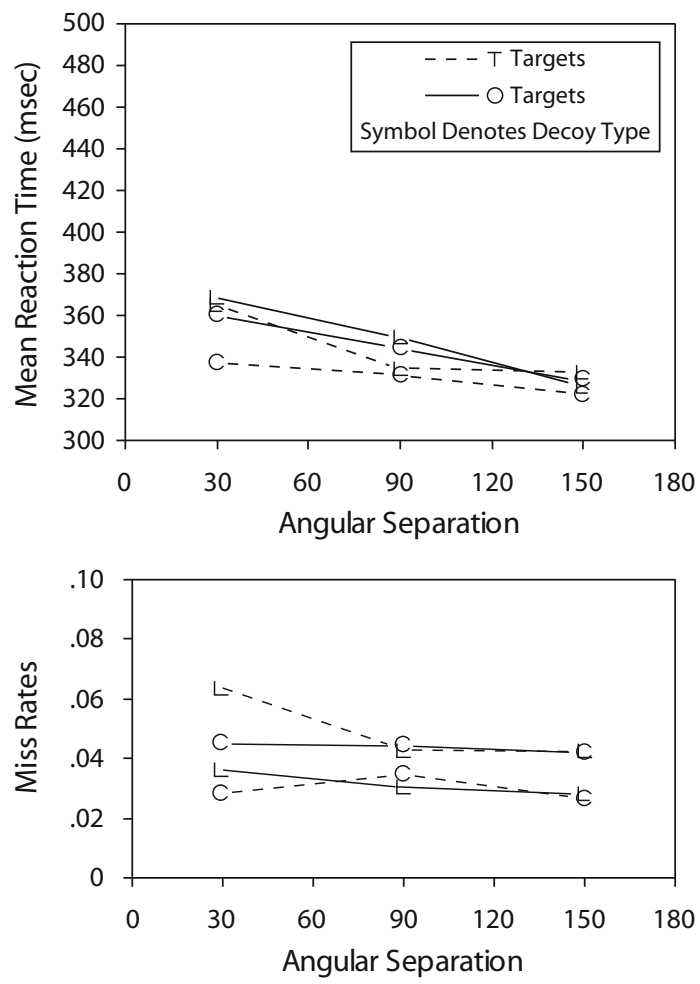

Figure 4. The top panel depicts mean correct reaction times from the go trials in Experiment 2. The type of target is depicted by the type of line $($ dashed $=0$, solid $=T$ ). The abscissa depicts the angular separation between the target and the decoy items. The bottom panel depicts the mean miss rate for each of the experimental conditions.

targets, an analysis yielded a significant main effect of separation $[F(2,38)=27.49, p<.001]$, but no reliable main effect of target-decoy similarity $[F(1,19)<1]$ and no reliable interaction $[F(2,38)<1]$. For the $\mathrm{O}$ targets, an analysis showed main effects of both target-decoy similarity $[F(1,19)=8.42, p<.01]$ and attended item separation $[F(2,38)=12.68, p<.001]$, but no reliable interaction $[F(2,38)=1.59$, n.s.]. The lack of a main effect of decoy form with $\mathrm{T}$ targets run counter to the predictions of the channel interaction account and is consistent with the predictions of the attentional weight account. For the $\mathrm{O}$ targets, there was a main effect of target-decoy similarity. However, an examination of Figure 4 reveals that the L decoys (low similarity) led to greater interference than did the $\mathrm{C}$ decoys (high similarity), a pattern opposite the predictions of the channel interaction account. Of most importance, neither target form showed a reliable interaction of separation with target-decoy similarity in RTs, confirming that target-decoy similarity had little effect on the strength of localized attentional interference in the present experiment.

Miss rates as a function of condition are shown in the bottom panel of Figure 4. An ANOVA similar to that performed on the RT data yielded only a main effect of target-decoy similarity $[F(1,19)=9.22, p<.01]$. An examination of Figure 4 reveals that miss rates were higher for target-decoy pairings with low similarity, again counter to the predictions of the channel interaction model. No other main effects or interactions approached statistical significance (all $F \mathrm{~s}<1$ ).

\section{GENERAL DISCUSSION}

Visual performance is often degraded when an observer is required to divide attention between a pair of spatially proximal objects. In the present experiments, we examined the mechanisms by which such interference is produced. The subjects' task in both experiments was to detect the presence of a designated target shape among a pair of color-cued items embedded within an array of gray filler items. Experiment 1 showed that the magnitude of localized interference was unaffected by similarity between the target stimulus and unattended filler items but was increased by similarity between the target and an attended decoy. In turn, Experiment 2 revealed that the strength of localized interference is determined not simply by similarity between the decoy and the target that are present within a given display but by the similarity between the decoy and either of two objects in the observer's target set, even one that is not actually present within the display.

In total, the results suggest a model in which (1) localized interference arises from competitive interactions between stimuli to be attended and (2) the competitive strength of an attended item is determined top down by its attentional weight. Conclusion 1 is motivated by the results of Experiment 1, which supported the reciprocal interference account over an alternative account suggesting that localized attentional interference arises from lapses in filtering of noise items when attended items are in close proximity. As was discussed in the introduction, a potential account of localized interference distinct from the reciprocal interaction hypothesis is that, as attended items are moved closer within the visual field, the observer is forced to relax the noise-filtering mechanisms that ordinarily support the attentional selection of an item. This might occur, for example, to reduce the effects of mutual suppression between the cued items. However, this hypothesis suggests that as the cued items move closer within the visual field and filtering is relaxed, filler items high in target similarity should produce greater interference than do filler items dissimilar to the target. Contrary to this prediction, the data showed no interaction of attended item separation with target-filler similarity, whereas an interaction between attended item separation and attended target-decoy similarity was observed.

Conclusion 2 arises from the finding that the strength of the localized attentional interference produced by an attended decoy is determined not simply by the similarity between the decoy and the target within a display, but by the similarity between the decoy and any item in the observer's target set. As has been noted, similarity-driven interference between attended items in Experiment 1 could be explained by either a channel interaction (Estes, 1972) or an attentional weight (Pashler, 1987) account. The channel interaction account holds that because the 
$\mathrm{T}$ target and the $\mathrm{L}$ decoy stimuli possess similar features, they are represented by a common set of feature-selective processing channels. Mutual interference between these stimuli was strong in the first experiment, then, because both were represented within the same limited pool of processing channels. In contrast, interference between the $\mathrm{T}$ target and the $\mathrm{O}$ decoy items was relatively modest because those shapes could be represented by processing channels selective for different features. According to the attentional weight account of the effect of target-decoy similarity effects, the $\mathrm{L}$ decoys led to greater interference because their relatively high similarity to the target gave them a higher attentional weighting than that given to the $\mathrm{C}$ decoys, making it more difficult to reject them as target candidates. In terms of the interactive competition hypothesis (e.g., Deco \& Rolls, 2004; Desimone \& Duncan, 1995; cf. Bundesen et al., 2005), the attentional set for the $\mathrm{T}$ targets results in a top-down biasing signal for features consistent with the target (e.g., vertical and horizontal line segments), increasing the competitive strength of the shapes that possess those features. Such top-down attentional bias has been shown to modulate localized attentional interference (e.g., Mounts, 2005; Mounts \& Gavett, 2004; Mounts \& Tomaselli, 2005), with increases in the attentional weight of a distractor leading to increases in the strength of localized interference.

This attentional weight account might also explain the main effect of target-filler similarity observed in Experiment 1. Although the filler items' form had no effect on the strength of localized attentional interference, the data did reveal a general slowing in RTs when the targets and the fillers were similar. One possibility is that this effect was the result of increased attentional competition between the fillers and the targets, albeit competition that was not spatially mediated. L- and T-shaped fillers, by virtue of their similarity to the T-shaped target, might have received greater attentional weighting than did C fillers. This would have allowed them to compete with the target more strongly than did the $\mathrm{C}$ fillers. Because filler items were distributed uniformly around the target, however, the effect of this competition would have been evident only as a general slowing in performance, unaffected by the spatial separation between the target and the attended decoy. Again, this is counter to the relaxed-filtering account examined in Experiment 1, since this interference or competition from the filler items was consistent across the various target-decoy separations tested.

Finally, two unexpected findings from Experiment 2 may provide further insight into the mechanisms producing localized attentional interference. First, stronger interference was observed, on average, with the $\mathrm{T}$ targets than with the $\mathrm{O}$ targets. Second, and perhaps more surprising, the $\mathrm{L}$ decoys produced stronger localized attentional interference with $\mathrm{O}$ targets than did the $\mathrm{C}$ decoys. Both of these findings might be attributable to the type of perceptual processing necessary to distinguish a $\mathrm{T}$ from an $\mathrm{L}$ and an $\mathrm{O}$ from a C. As others have noted, the T-L discrimination involves distinguishing the relative spatial locations of a common set of features (Huang \& Pashler, 2005; Wolfe, 1998), whereas the O-C discrimination involves identify- ing the presence or absence of a feature (e.g., a gap or closure; Treisman \& Souther, 1985). The data suggest that the spatial arrangement discrimination involved in distinguishing a $\mathrm{T}$ from an $\mathrm{L}$ may be more resource intensive (Huang \& Pashler, 2005) than is the feature detection task required to discriminate an $\mathrm{O}$ from a $\mathrm{C}$. This may leave the O-shaped targets less susceptible to localized attentional interference than are the T-shaped target stimuli. Further empirical work will be required to test these suggestions.

\section{AUTHOR NOTE}

This work was supported in part by Grant EY1368701 from the National Eye Institute to J.R.W.M. Address correspondence to J. R. W. Mounts, Department of Psychology, State University of New York, Geneseo, NY 14454 (e-mail: mounts@geneseo.edu).

\section{REFERENCES}

BAHCALl, D. O., \& Kowler, E. (1999). Attentional interference at small spatial separations. Vision Research, 39, 71-86.

Bundesen, C., Habekost, T., \& Kyllingsbek, S. (2005). A neural theory of visual attention: Bridging cognition and neurophysiology. Psychological Review, 112, 291-328.

Caputo, G., \& Guerra, S. (1998). Attentional selection by distractor suppression. Vision Research, 38, 669-689.

Cave, K. R. (1999). The FeatureGate model of visual selection. Psychological Research, 62, 182-194.

Cave, K. R., \& Zimmerman, J. M. (1997). Flexibility in spatial attention before and after practice. Psychological Science, 8, 399-403.

Cutzu, F., \& Tsotsos, J. K. (2003). The selective tuning model of attention: Psychophysical evidence for a suppressive annulus around an attended item. Vision Research, 43, 205-219.

Deco, G., \& Rolls, E. T. (2004). A neurodynamical cortical model of visual attention and invariant object recognition. Vision Research, 44, 621-642.

Desimone, R., \& Duncan, J. (1995). Neural mechanisms of selective visual attention. Annual Review of Neuroscience, 18, 193-222.

Dosher, B. A., \& LU, Z.-L. (2000). Noise exclusion in spatial attention. Psychological Science, 11, 139-146.

DunCan, J. (1984). Selective attention and the organization of visual information. Journal of Experimental Psychology: General, 113, 501-517.

Duncan, J., \& Humphreys, G. W. (1989). Visual search and stimulus similarity. Psychological Review, 96, 433-458.

Egly, R., Driver, J., \& Rafal, R. D. (1994). Shifting visual attention between objects and locations: Evidence from normal and parietal lesion subjects. Journal of Experimental Psychology: General, 123, 161-177.

Eriksen, C. W., \& Hoffman, J. E. (1973). The extent of processing of noise elements during selective encoding from visual displays. Perception \& Psychophysics, 14, 155-160.

Estes, W. K. (1972). Interactions of signal and background variables in visual processing. Perception \& Psychophysics, 12, 278-286.

Hamker, F. H. (2004). A dynamic model of how feature cues guide spatial attention. Vision Research, 44, 501-521.

HOFFMAN, J. E. (1978). Search through a sequentially presented visual display. Perception \& Psychophysics, 23, 1-11.

HuANG, L., \& Pashler, H. (2005). Attention capacity and task difficulty in visual search. Cognition, 94, B101-B111.

IshiHARA, S. (1989). Tests for colour-blindness. Tokyo: Kanehara.

Kastner, S., De Weerd, P., Pinsk, M. A., Elizondo, M. I., Desimone, R., \& UNGERLEIDER, L. G. (2001). Modulation of sensory suppression: Implications for receptive field sizes in the human visual cortex. Journal of Neurophysiology, 86, 1398-1411.

Kim, M.-S., \& CAVE, K. R. (2001). Perceptual grouping via spatial selection in a focused-attention task. Vision Research, 41, 611-624.

Kristjánsson, Á., \& NAKAYAMA, K. (2002). The attentional blink in space and time. Vision Research, 42, 2039-2050.

Lu, J., \& ItTI, L. (2005). Perceptual consequences of feature-based attention. Journal of Vision, 5, 622-631. 
Lu, Z.-L., \& Dosher, B. A. (1998). External noise distinguishes attention mechanisms. Vision Research, 38, 1183-1198.

Lu, Z.-L., \& Dosher, B. A. (2004). Spatial attention excludes external noise without changing the spatial frequency tuning of the perceptual template. Journal of Vision, 4, 955-966.

LuCK, S. J., \& BEACH, N. J. (1998). Visual attention and the binding problem: A neurophysiological perspective. In R. D. Wright (Ed.), Visual attention (pp. 455-478). New York: Oxford University Press.

Luck, S. J., Girelli, M., McDermott, M. T., \& Ford, M. A. (1997) Bridging the gap between monkey neurophysiology and human perception: An ambiguity resolution theory of visual selective attention. Cognitive Psychology, 33, 64-87.

McCarley, J. S., \& Mounts, J. R. W. (2007). Localized attentional interference affects object individuation, not feature detection. Perception, 36, 17-32.

McCarley, J. S., Mounts, J. R. W., \& Kramer, A. F. (2004). Agerelated differences in localized attentional interference. Psychology \& Aging, 19, 203-210.

Moran, J., \& Desimone, R. (1985). Selective attention gates visual processing in the extrastriate cortex. Science, 229, 782-784.

Mounts, J. R. W. (2000a). Attentional capture by abrupt onsets and feature singletons produces inhibitory surrounds. Perception \& Psychophysics, 62, 1485-1493.

Mounts, J. R. W. (2000b). Evidence for suppressive mechanisms in attentional selection: Feature singletons produce inhibitory surrounds Perception \& Psychophysics, 62, 969-983.

Mounts, J. R. W. (2005). Attentional selection: A salience-based competition for representation. Perception \& Psychophysics, 67, 1190-1198.

Mounts, J. R. W., \& Gavett, B. E. (2004). The role of salience in localized attentional interference. Vision Research, 44, 1575-1588.

Mounts, J. R. W., \& Tomaselli, R. G. (2005). Competition for representation is mediated by relative attentional salience. Acta Psychologica, 118, 261-275

PAshler, H. (1987). Target-distractor discriminability in visual search. Perception \& Psychophysics, 41, 285-292.

Posner, M. I. (1980). Orienting of attention. Quarterly Journal of Experimental Psychology, 32, 3-25.

REYNOLDS, J. H., \& DESIMONE, R. (1999). The role of neural mechanisms of attention in solving the binding problem. Neuron, 24, 19-29.

REYNOLDS, J. H., \& DESIMONE, R. (2003). Interacting roles of attention and visual salience in V4. Neuron, 37, 853-863.

Saenz, M., Buracas, G. T., \& Boynton, G. M. (2002). Global effects of feature-based attention in human visual cortex. Nature Neuroscience, 5, 631-632.

Theeuwes, J., Kramer, A. F., \& Kingstone, A. (2004). Attentional capture modulates perceptual sensitivity. Psychonomic Bulletin \& Review, 11, 551-554.

Treisman, A. (1996). The binding problem. Current Opinion in Neurobiology, 6, 171-178.

Treisman, A., \& Souther, J. (1985). Search asymmetry: A diagnostic for preattentive processing of separable features. Journal of Experimental Psychology: General, 114, 285-310.

Treue, S., \& Martínez Trujillo, J. C. (1999). Feature-based attention influences motion processing gain in macaque visual cortex. Nature, 399, 575-579.

Tsotsos, J. K., Culhane, S. M., Wai, W. Y. K., LaI, Y., Davis, N., \& Nuflo, F. (1995). Modeling visual attention via selective tuning. Artificial Intelligence, 78, 507-545.

Turatto, M., \& Galfano, G. (2001). Attentional capture by color without any relevant attentional set. Perception \& Psychophysics, 63, 286-297.

VECERA, S. P. (2000). Toward a biased competition account of objectbased segregation and attention. Brain \& Mind, 1, 353-384.

Wolfe, J. M. (1998). Visual search. In H. Pashler (Ed.), Attention (pp. 13-73). East Sussex, U.K.: Psychology Press.

YEH, Y.-Y., \& ERIKSEN, C. W. (1984). Name codes and features in the discrimination of letter forms. Perception \& Psychophysics, 36, 225-233.

\section{NOTES}

1. The selective tuning model of Tsotsos et al. (1995) also incorporates an inhibitory feedback mechanism that creates a region of inhibition surrounding the selected object.

2 . Four different values of filler similarity were chosen because, a priori, it was not clear what aspects of similarity would be critical. Thus, we chose fillers that (1) were possible target values (canonical and $180^{\circ} \mathrm{Ts}$ ), (2) possessed the same shape as the target, but in a nontarget orientation $\left(90^{\circ}\right.$ and $\left.180^{\circ} \mathrm{Ts}\right),(3)$ possessed the same features as the target, but in a different spatial arrangement (Ls), and (4) possessed features distinct from the target features (Cs).

(Manuscript received September 28, 2005; revision accepted for publication April 14, 2006.) 